\title{
Barriers to Optimal Maternal and Child Feeding Practices in Pastoralist Areas of Somali Region, Eastern Ethiopia: A Qualitative Study
}

\author{
Abdulahi Haji Abas*, Ahmed Tahir Ahmed, Abdifatah Elmi Farah, Girma Taddese Wedajo \\ Department of Public Health, College of Medicine and Health Science, Jigjiga University, Jigjiga, Ethiopia \\ Email: *cabasaw@gmail.com
}

How to cite this paper: Abas, A.H., Ahmed, A.T., Farah, A.E. and Wedajo, G.T. (2020) Barriers to Optimal Maternal and Child Feeding Practices in Pastoralist Areas of Somali Region, Eastern Ethiopia: A Qualitative Study. Food and Nutrition Sciences, 11, 541-561.

https://doi.org/10.4236/fns.2020.116038

Received: February 27, 2020

Accepted: June 15, 2020

Published: June 18, 2020

Copyright $\odot 2020$ by author(s) and Scientific Research Publishing Inc. This work is licensed under the Creative Commons Attribution International License (CC BY 4.0).

http://creativecommons.org/licenses/by/4.0/

(c) (i) Open Access

\begin{abstract}
Maternal and child nutrition is critical for child health and survival. Appropriate feeding practices are of fundamental importance for health, nutrition, survival and development of infants and children. In pastoral areas of Ethiopia, barriers related to culture, knowledge, social norms, beliefs, behaviors, decision making in the household and burden of other responsibilities contribute to nutritional status of women and children to deteriorate. Policies and strategies are recommending assessment of barriers for designing programs and interventions to improve maternal and child nutrition practices. This study is aimed to assess barriers of optimal maternal and child feeding practices in Pastoralist areas of Somali region, Eastern Ethiopia. A qualitative community based research method was used involving 17 focus group discussions and 20 in-depth interviews with mothers, grandmothers, health professionals and religious leaders from three districts. Data from FGDs and interviews were transcribed and coded. The agreed upon codes were synthesized and grouped into exhaustive categories. The categories were then merged into themes representing the most common barriers on maternal and child feeding practice that emerged from the FGDs and interviews. We found that traditional beliefs, myths, culture custodian influence, low accessibility and availability of nutritional foods, pattern and burden of other responsibilities, poor knowledge and health seeking behavior, perceived milk insufficiency as main barriers for optimal maternal and child feeding practices. Barriers on optimal maternal and child feeding are very common in the study areas. More work needs to be done to strengthen community-based nutrition with strong social behavioral change communication with emphasis on age-specific counselling on maternal and child nutrition at health facilities, during antenatal and early postnatal visits in the study areas through different media channels, particularly, Somali Region TV and FMs to promote optimal nutri-
\end{abstract}


tion in the region.

\section{Keywords}

Barriers, Maternal, Child, Nutrition, Somali Region, Eastern Ethiopia

\section{Introduction}

Maternal and child nutrition is critical for child health and survival. Optimal feeding practices are of fundamental importance for health, nutrition, survival and development of infants and children. Maternal nutrition is critical to both mother and child [1]. Poor nutritional status before and during pregnancy is been associated with intrauterine growth retardation (IUGR), low birth weight (LBW) and premature delivery conditions. The critical window for improving child nutrition is from pregnancy through the first 24 months of life [2].

Optimal maternal nutrition during the first 1000 days is critical from conception through the first 6 months of life to improve the nutritional status of the woman and infant and reduce the risk of adverse birth outcomes such as low birthweight and preterm birth [3] [4]. Several barriers exist that hinder adequate dietary intake among pregnant and lactating women. Food intake during pregnancy and lactation is largely driven by personal preferences and cravings. Other factors include food avoidance due to cultural beliefs or food taboos, perceived appropriateness of foods for pregnancy or lactation/postpartum, economic constraints and beliefs surrounding pregnancy physiology. Some studies indicated lack of knowledge and beliefs as main barriers for optimal maternal nutrition [5].

Food avoidance was also identified as a barrier for maternal nutrition; food of high nutrient value was avoided during pregnancy due to a range of cultural taboos and misinformation [6]. Food avoidance can also stem from beliefs of ill-effects during labor and delivery caused by barriers to maternal diet during pregnancy and lactation [7].

Another barrier was the lack of understanding the physiological state of pregnancy and the fetal development process in relation to maternal diet. Study revealed that mothers, community leaders, and health promoters related poor nutrition during pregnancy to low birthweight or intrauterine growth restriction because they attributed the child's health to "divine will" [8]. Similarly, in Ethiopia, study results reported that women thought the health of the baby is determined by God [9]. Few studies cited beliefs that fetus and food were both located in the stomach, and therefore if women ate too much food the baby would not have room to grow [10].

Women also relayed intentionally eating less food during pregnancy due to fear of having a large baby and long, difficult labor. The practice of intentionally eating less or "eating down" during pregnancy for fear of delivering a large baby 
and enduring long, painful labor was cited in several countries, including Senegal, Nepal, Laos, India, Japan, Pakistan, Indonesia, and Burkina Faso [9] [11]. Economic constraints are also reported as a major barrier to obtain food in Bangladesh, Indonesia, Burkina Faso, Egypt, Ethiopia, India, Kenya, Nepal, Nigeria, Pakistan, and Tanzania (e.g. milk), fish, chicken, eggs, and red meat [12].

Child feeding practices comprise of both breastfeeding and complementary feeding practices [13]. Globally, only a few children are receiving nutritionally diversified and adequate foods. In many countries, less than $25 \%$ of infants aged 6 - 23 months meet the criteria for dietary diversity and feeding frequency. This problem affects more in developing countries [14]. Sub-optimal breastfeeding in the first 6 months of life results in 1.4 million deaths and $10 \%$ of the disease burden in children younger than 5 years [15] [16] [17]. Early initiation of breastfeeding results in better establishment of exclusive breastfeeding practices and continued breastfeeding [5] [18] [19].

Even though breastfeeding is a natural process, it is reported to be influenced by different socio-cultural factors, customs, values, and behaviors [5]. Studies show that cultural behaviors related to breastfeeding may be damaging, harmless, or beneficial to the optimal breastfeeding practices [20].

The harmful cultural traits reported as barriers for optimal breastfeeding practices include: giving pre-lacteal feeds, discarding colostrum and avoiding breastfeeding after quarreling out of fear of bad blood entering the milk which later may affect the child [21] [22] [23] [24].

In other situations, lack of support from family members or health care professionals, peer pressure, mothers' body image, the role of women in the reproduction process, and pressure to use artificial feeding have led to early cessation of EBF and breastfeeding [25] [26] [27].

Barriers for optimal complementary feedings are also indicated by various studies, socio cultural factors and traditional practices influence the practice of introducing early complementary feeding [28]. Caregivers lack the knowledge about appropriate complementary feeding and quantity and quality of foods [29]. Lack of knowledge and understanding among health workers is significantly associated with poor child complementary feeding practices [30]. Low income and poor household food security are important factors in nutritional outcomes of infants and young children [30].

Globally, barriers on optimal practice for maternal and child nutrition have been the subject of various research studies. Studies have reported poverty [31], poor knowledge [32] [33] [34] [35], influence of culture custodians, lack of decision-making power in the household [36], patterns and burden of other work responsibilities [37], poor maternal dietary intake and perceived milk insufficiency [33] [36] [38] [39] as barriers for optimal maternal and child nutrition in general.

Poor breastfeeding and complementary feeding practices have been widely documented in Ethiopia. Only 58\% of infants less than six months old were ex- 
clusively breastfed, $48.4 \%$ of infants 6 - 8 months were breastfed and received appropriate complementary feeding, and $51.5 \%$ of neonates were breastfed within one hour of delivery [40]. In Ethiopia, mothers give herbal extracts to their children, discarded colostrum, and introduced complementary feeding as early as 3 months [20].

In pastoral areas, barriers related to cultural, knowledge, social norms, beliefs, behaviors, decision making in the household, burden of other responsibilities and availability of nutrients are making nutritional status of women and children deteriorate. As result, dietary diversification is decreasing and declining of milk production leaves most households with milk available only during the rainy season [41]. Surveys conducted in Ethiopia also show that in the Somali region only $4.3 \%$ and $0.8 \%$ of children 6 - 23 months consumed four or more food groups and had Minimum Acceptable Diet (MAD) respectively [42]. The high prevalence of low birth weight (LBW) at $14.8 \%$ in the Somali Region, higher than the national average of $10.8 \%$, has its root in poor nutritional status of women both before and during pregnancy as well as inadequate weight gain during pregnancy. According to Ethiopian Demographic and Health Survey EDHS 2016, maternal under nutrition (BMI < 18.5) was 32.7\% among women in Somali region compared to the national rate of $27 \%$. These figures translate to higher levels of low birth weight which contributes to intergenerational malnutrition [40].

Different quantitative studies have reported barriers on maternal and child feeding practice in Ethiopia. The barriers include cultural, knowledge, social norms, beliefs, decision making in the household, burden of other responsibilities and availability of nutrients. Few qualitative studies have explored barriers for maternal and child feeding practice. As far as our knowledge is concerned, both qualitative and quantitative studies are limited or not existing in Somali region. Thus, this study is aimed to assess barriers to optimal maternal and child feeding practice in pastoralist areas of Somali region of Ethiopia.

\section{Methods}

Qualitative Community based was conducted from 12th of July 2018 to 10th of August, 2018, through focus group discussions (FGDs) and in-depth interviews with caregivers of infants aged 0 - 24 months, religious leaders and health professionals. The study was conducted in Dagahbur, Gursum and Goljano districts of Fafan Jarar zones Somali Regional State, Eastern Ethiopia. The purpose of the study was to explore barriers to optimal maternal and child feeding practice in the selected sites.

\subsection{Study Areas and Recruitment}

The study was conducted in Goljano and Gursum districts of Fafan zone and Dagahbur district of Jarar zone, Somali region, eastern Ethiopia, Dagahbur is relatively urban and $(180 \mathrm{~km})$ from Jigjiga, capital city of Somali region. 
Data were collected by three nurses and one nutritionist for each district; data collection process was supervised by the principal investigators. The researchers approached the local council leaders of six selected villages and sub-villages to initiate the data collection process. The village leaders who are the responsible for the public records about residents in the village provided researchers with information about households with pregnant women and infants/ children in the age-range of 0 - 24 months.

The inclusion criteria were pregnant women, caretakers for children aged 0 24 months, health professionals and religious leaders in selected sites. We preferred these categories from the villages, particularly primary care givers because they are more likely to be able to recall maternal and child feeding practices. We explained the objectives of the study and asked the mothers/caregivers and religious leaders to give consent to participate in the study. Written consent was obtained from the study participants who agreed and were willing to participate.

The focus group discussions were conducted in various settings preferred by the group members, including the health center, health posts, villages offices, community meeting halls, under trees and in a classroom. Each focus group was comprised of 8 - 11 mothers and the discussions lasted for one to one and a half hour. In addition to this, in-depth interview was conducted with religious leaders and health professionals. The FGDs on each day was guided by one of the researchers, using the interview guide. The proceedings of the FGD and in-depth interview were recorded using a voice tape-recorder, and detailed notes were taken by the research teams.

\subsection{Data Collection}

An FGD and interview guide with written questions and probes were used during the data collection. It included questions like the maternal nutrition during pregnancy and breastfeeding, meaning of infant and young child feeding practices and personal experiences with maternal nutrition, exclusive breastfeeding, community influences, and practices regarding colostrum, insufficient milk, signs of a hungry child, introduction of complementary feeding, contents, information given during delivery, complementary feeding, challenges, and suggestions to improve the practice of maternal and child nutrition practice in the community.

The focus group discussion was conducted in Somali and facilitated by trained researchers with experience in FGD. The facilitator is a native Ethiopian and speaks Somali. All participants were identified by number and not by name, and before the start of each FGD the facilitator discussed with the participants the issue of confidentiality. During discussions, hesitant participants were reassured of anonymity which created a comfortable environment for them to talk.

Participants were also informed that the discussions and interview would be tape-recorded for later use by the researcher. Further, field notes were taken by research assistants which helped the research team in identifying new informa- 
tion relevant to the study. The field notes were used for triangulation. After each FGD and interview, there was a debriefing where the researcher and the nurses discussed the important themes and newly emerging themes.

\subsection{Data Analysis}

A thematic analysis was performed according to six steps as described by Braun and Clarke [43]. The first step was familiarization with the data whereby the FGDs and interviews were transcribed verbatim and translated to English by the facilitator. All the transcribed FGDs and interviews were read by two investigators who did the data analysis. During the second step, the investigators developed initial codes individually and later compared them with each other. The developed codes were refined until there was no new code that emerged. The third step was searching for themes. A matrix table was used to list the codes, and all the codes that were related were sorted and listed into one theme.

The last three steps involved reviewing and refining the themes and report writing. Themes representing the most common barriers on maternal and child feeding practices that emerged from the FGDs and interviews were refined. The results presented below are organized in a way that shows themes explaining the main barriers for appropriate nutrition practice.

\subsection{Ethical Considerations}

The study was approved by Jigjiga University ethical committee. A letter of ethical approval was obtained Jigjiga University before commencing data collection. Permission letter was also provided to district health offices. Participants were well-oriented about the purpose and procedure of data collection, and that confidentiality and privacy will be ensured.

\section{Result}

The results are presented in three main themes; barriers to optimal diet during pregnancy, and the second comprises factors that enable or facilitate optimal MIYCN practices. These two main themes are also broken down into sub-themes; maternal, infant and young child nutrition.

\subsection{Barriers to Optimal Diet during Pregnancy}

There are four sub-themes generated from FGDs and key interviews data that explain barriers to optimal maternal nutrition during pregnancy, the sub-themes are; traditional beliefs and myths, poor knowledge on maternal nutrition, poor accessibility of health services and nutrients, influence of culture custodians and burden of other responsibility.

\subsubsection{Traditional Beliefs, Myths, and Misconceptions Surrounding Pregnancy Physiology}

This study revealed that mothers belief that pregnancy is associated with diseases and difficulties. So, intake of nutrients decreases. The study also revealed that 
mother and grandmother belief if pregnant mother increases intake of foods during pregnancy fetus will be larger than the birth canal.

"I usually limit food and nutrient intake during pregnancy due to vomiting and nausea; at same time I also believe if I increase intake of food during pregnancy the fetus will be bigger to pass through the birth canal' [Dagahbur town, Mother 3].

This practice of limiting intake of food and nutrients during pregnancy is reinforced by important and influential members of the family such as grandmothers, as indicated below.

"If intake of food increases, this causes the fetus larger than the birth canal, and the child will need more power than mother's energy (wuu ka tan badanayaa ilmuhu wakhtiga dhalmada). Therefore, we recommend mothers to limit intake of foods until 7 months are completed in which pregnant mothers could take food' [Fafan, Grandmother 10].

This study also discovered that mothers and grandmothers associate intake of Iron tablet with pregnancy difficulty, especially larger fetus and complication during delivery which they think will expose the pregnant mother to caesarian-section, if intake of iron is consumed as recommended by the health professional.

"Iron supplementation is said to be associated with larger fetus, but I do not take iron supplementation during pregnancy unless I am losing power and having headache" [Goljano, Sandmadhedhe, Mother 4].

This misconception of limiting intake of Iron supplementation or taking Iron supplementation when mother feels headache and fatigue is also recommended by influential members in the households, such as grandmothers as echoed below.

"I recommend my daughter to take the iron supplementation from health facility when she feels headache and fatigue (awoodayadu dhacsantahay), and iron is given for improvement. I do not advise mothers to take Iron tablet while they are in good health" [Fafan, Grandmother 10].

\subsubsection{Poor Knowledge on Optimal Diets for Pregnant Mothers}

The participants reported inappropriate practices on optimal diets during pregnancy and these were mostly stemming from lack of knowledge on increased requirement of diets during pregnancy. Mothers had low knowledge on extra food needed during pregnancy. However, if there is any change on appetite of pregnant mother on intake of food, they do not think to insist taking extra food as indicated below.

"Pregnancy is associated with decreased intake of food especially during first three months (first trimester) because I do not take food properly due to pregnancy related nausea and vomiting. If I am not able to take the food I should not bother and I should wait until these symptoms decreases after first trimester, we usually say pregnant women neither eat the food nor miss to eat the food" [mana qado mana qaado) (Dhagahbur city, Mother 2]. 
Mothers have also low knowledge when it comes to type of diet to eat and low knowledge level of about optimal diets needed during pregnancy. Pregnant mothers had knowledge only that animal source foods are needed during pregnancy, but they do not know other components of balanced diets needed during pregnancy such as fruits, legumes and vegetables.

"We should take animal source foods during pregnancy if available, animal source foods are nutritious during pregnancy. So, I don't know other foods which are good for pregnant mothers as animal food" [Goljano, Sandmadhele, Mother 3].

Grandmothers had also low knowledge level on requirement of extra food during pregnancy; they do not know extra food is needed due to pregnancy hence they do not recommend mothers to take more foods during pregnancy.

"its natural thing, one day she may require certain type of foods, other day another food, the other day she will stop eating, we usually support the mother according to her need; if she does not need any food for that day we do not prepare for them. My recommendation during pregnancy depends on her, if she needs, we prepare type of food she prefers" [Dhagahbur, Grandmother 2].

Religious leaders also had low knowledge on requirement of extra food for pregnant women. In addition to this, religious leaders do not have platform to recommend mothers on nutrition; they do not even know whether they need or not. Fathers had low knowledge on this requirement for extra food for pregnant women; fathers are not aware of what mothers are eating during pregnancy. Therefore, they have no role on type of food and extent of intake for mothers during pregnancy.

"I have no knowledge on extra requirement of diets for pregnant women; I have no information and there is no platform I know to recommend dietary intake for pregnant women in my kebele" [Dagahbur, religious leader].

"I do not have any knowledge what my wife is taking during pregnancy, I am a busy pastoralist with the livestock the whole day and come home late at night, therefore, I have no any information on what my wife eats at day and at night" [Gursum, Gorey, Father 2].

\subsubsection{Low Accessibility and Affordability of Diets and Health Services} Accessibility and availability of different foods in some kebeles is another barrier for pregnant mothers to have fully diversified diets needed during pregnancy. This accessibility barrier is also indicated by grandmothers in pastoralist kebeles,

"There is scarcity of fruit, vegetables and legumes here; we have only animal source foods for pregnant mothers to eat which also become scarce now days due to recurrent droughts. Sometimes, banana and onion may be available at market in Goljano town but, it's rare for mothers to have these foods from Goljano, unless they go for other purposes" [Goljano, Sanmadhedho, Mothers 3].

"I recommend mothers to eat animal source foods because there is no other nutrient in the kebeles. There is no legumes, fruits and vegetables available in the 
kebeles, if we need we should go to Dagahbur which is not always possible for pastoralist pregnant women" [Dagahbur, Agasur, Grandmother 5].

Some kebeles in the study areas have no health facility and community should go certain kilometers to have the service, therefore, pregnant mothers in these kebeles do not have access to Iron supplementation, counselling, screening and treatment for acute malnutrition during pregnancy.

"We don't have health facility in this kebele, therefore, we go to other nearby health facilities to have the service, only if I am seriously sick. The nearest health facility is around five kilometers from this kebele. In addition to this, I am pastoralist so I am busy with my livestock, I am taken to nearby health facility if I am ill with a disease" [Goljan, sanmadhede, Mother 4, FGD].

\subsubsection{Pattern and Burden of Other Responsibilities during Pregnancy}

In pastoralist areas, support from family, particularly husband is rare because the family is busy with livestock and pregnant women are busy in livestock rearing. In addition, some of the family members see as inappropriate culturally if the husband supports pregnant women for household activities. Thus, pregnant women feel shame to ask support and also the husband may refuse as depicted below. This culture of avoiding supporting pregnant mothers is deeply rooted in the community; even grandmothers avoid recommending fathers to support pregnant women in some households.

"Husband asks you to avail everything for him instead of supporting you, even if he agrees and tries to support you other husbands and neighbors will say to him that he is doing responsibilities and chores to be done by his wife and he is 'QORQODE a man who is always with women (supporting food preparation)" [Gursum, fafan, Mother 3, FGD].

"I do not recommend mothers to ask support from her husband since neighbors would see it as offensive to her, nor would husband try to support domestic activities in fear off negativity from other neighboring husbands" (Grandmother 4, Goljano, FGD].

Participants from fathers group reported that they are busy with livestock; searching for pasture and water, fathers are not aware off nutrition intake for pregnant mothers.

"I do not support my wife with any household activities. I am busy with livestock, so, my wife brings the water wherever she can find in top of other demotic work' [Father 10, sanmadheedhe, Goljano woreda, FGD].

\subsection{Barrier to Optimal Diets during Lactation}

\subsubsection{Poor Knowledge about Optimal Diets during Lactation}

The participants reported that increased intake of food during lactation, in Somali culture, some husbands usually slaughter animal immediately after delivery. But, the participants revealed that lactating mothers eat mostly foods from cereals in which animal foods are added rarely due to impact of recurrent drought in pastoralist areas. The participants had low knowledge levels on type of diets to 
be taken during lactation, particularly, fruit, vegetables, and legumes.

"I encourage lactating mother to eat more 'Mushal', food made from cereal with milk and reduce intake fruits and vegetables since fruits and vegetable induce diarrhea" [ Grandmothers 2, Dagahbur woreda, FGD].

The participants mentioned that animal foods are very nutritious if available and fruits, while legumes and vegetables are not needed at this period, this was one of the indicators of low knowledge on diversified foods. Others indicated that having animal product is important than vegetables and legumes, participants also pointed out low interest for vegetables and legumes claiming that these diets do not increase mother's weight during lactation.

"Mothers should regain the weight they lost during pregnancy. They should eat more food from animal source, fruits, vegetables and legumes do not assist mothers to regain the weight they lost during pregnancy" [Mothers 3, Gursum, $F G D$.

\subsubsection{Low Health Seeking Behavior}

Health professional at health facilities reported that mothers do not come back to health facility after delivery to receive counselling on diets to be taken; they come back only if they get sick or their infant gets sick. Health professional also reported that their outreach visits are very minimum, complaining that the limited number of health workers in the facilities and instead focus only static health service delivery.

"It's rare to see mothers coming back to health facility to receive postnatal care, they come back mostly when their infants are sick or they feel not good" [Health professional, Gursum, interview].

"We are few in number in this health facility, we are always busy with high number of clients in this facility, thus, we do not go out to give outreach service for the community consistently" [Health professional, Gorey, interview]

\subsubsection{Pattern and Burden of Other Responsibilities during Lactation}

Some of the mothers reported that they heard about availability of postnatal services but failed to attend the services due to lack of time because of work and other responsibilities. Participants reported that they are busy with feeding their families, rearing livestock and fetching water for families and livestock since they are pastoralist.

"We have no time for going back to health facility to receive postnatal care services, nor do we have a time to look for taking diversified food during lactation. We are pastoralist and we should spent time making sure that our livestock is getting enough pasture and water. In addition, we should also be busy for family to be fed well' [Mother 8, Sanmadheedhe, Goljano, FGD].

Other participants reported that they only get rest for first 40 days after the delivery. After that they should start looking after their livestock, farms and families. Lactating mothers do not cook food for themselves separately.

"I get rest for the first 40 days of birth; after that no one will support us. So, we 
should be busy with the family, livestock and farms" [Mother 7, Gorey, FGD].

\subsection{Barriers on Optimal Child Feeding Practices}

\subsubsection{Low Knowledge and Attitude about Breastfeeding and Complementary Feeding}

Majority of the participants in the FGDs reported that they gave holy water and fax (sugary water) to children immediately after delivery, until breastmilk comes to the breast naturally at least after three days. Some of the grandmothers also believe that it's natural for some women not to produce milk at first time and such women should wait some time.

"Breastmilk comes to my breast after three days. Therefore, I have to prepare 'fax' to feed the infant after the holy water is given. After three days, milk comes and I start giving breastmilk' [Mother 6, sandmadeda, Goljano woreda].

"I don't practice skin to skin for infants immediately after the delivery, I recommend mothers to prepare 'fax' and any other animal milk she could afford to give the baby until the milk comes, after three days mostly" (Grandmothers 10, Agasur, Dagahbur woreda).

The duration of exclusive breastfeeding was short due to the common practices of giving infants water and pre-lacteal feeds, so, majority of participants practiced giving water for infants less than six months. These study participants believed that their babies needed water by claiming that breastmilk is too hot and comes through blood vessels. Water should be given to the infants in order to cool down.

"Giving water has no time; we start giving water at early age because the milk is hot since human milk comes through blood vessels" [Mother 11, Dagahbur].

Furthermore, some of the participants reported that water should be given to male infants because male genitalia make the infants thirsty and more water is needed. Some of the participants also reported difficulty in breastfeeding due to a perception that there is not enough breastmilk. As a result, some mothers in the FGDs and interviews pointed out that they have started food or milk at early stage before six months.

"I recommend water to be given for infants at every stage before six months, particularly, male infants, because male infants need more water due to male genitalia" [Mother 2, Dagahbur].

"I started feeding my infant a powder milk while he was two months because my breastmilk was not enough for the baby. Now days all mothers are giving other milk and foods before the child is six months saying that breastmilk is not enough" [Mother 8, Dagahle, Gursum woreda].

"I started food, particularly animal source food for child to grow well otherwise the child would not grow well and would be undernourished; Uurbasaas" [Mother 6, Dagahle, Gursum woreda].

Furthermore, Mothers explained a children's cry indicates that they are not satisfied during or after breastfeeding. This, combined with mothers' perceived inability to produce enough milk led them to introduce foods generally within 
the first 2 to 3 months of life. Mothers felt encouraged in this practice when children stopped crying after being given food. When the child cries, the child is hungry was frequently voiced as well as the belief that giving sugary water or animal milk makes children calm. Several mothers discussed this practice.

"When my child cries, I usually start breastfeeding but if the child is not satisfied and stop cryin, then I start giving milk powder to satisfy" [Mother 9, Dagahbur].

"If the baby cries and not satisfied by breastmilk we recommend mothers to give food or animal milk other than breastmilk because breastmilk is not enough" [Grandmother 12, sanmadeda, Goljano woreda].

In general, porridge was introduced to children, followed by food the family eats (Rice, Lahoh, Pasta, Shuro). Women felt that the children's crying indicates when they are ready for complementary foods. As mothers explained they start feeding their children Shuro at any age. The participants further pointed out that they start food when the child is not satisfied by the breastmilk only. In addition, mothers interpret a child's cry as desire to eat family food.

Maize and Wheat flours were often used to make Shuro and lahoh (Injero); some of these foods take at least 24 hours to be ready for the family. Most often, Shuro consisted of Maize flour, milk and water, though access to other flours varied depending on location. In urban area, legumes are also mixed with other flours. There is early introduction of complementary feeding as indicated by the participants. Moreover, some participants reported late introduction of complementary feeding. Caretakers indicated that they started animal milk as early as possible but food at 12 months as per below statement.

"I check the child whether she can take or not a soft and semi-solid food at four months, I should know whether my child could take the food as early as possible otherwise the child will not grow well' [Mother 6, Goljano woreda].

"I never started food for my children before one year, I usually start soft foods at first birthday" [Mother 4, Agasur, Dagahbur woreda].

On top of this, lack of knowledge on how to enrich complementary food was also another barrier to optimal complementary feeding. Many mothers reported that they don't know how to add other food items including carrot and or eggs. As cereals are mostly used for complementary food preparation, participants indicated that they only add milk.

"I use flour made from either wheat or maize, we use this flour for making Shuro for the household, including the children. We eat this Shuro with milk for whole family if available. If there is scarcity of milk, we give Shuro with little milk for the children only" [Mother 8, Dagahle, Gursum woreda].

Additionally, many mothers indicated that breastfeeding while pregnant would negatively impact the health of the child, and pregnancy was a common reason for cessation of breastfeeding. Lack of knowledge on frequency of complementary feeding was also identified in this research. Majority of mothers do not know how many times children should be fed per day. 
"I stopped breastfeeding of my child after becoming pregnant for fear of spoiling the health of my child because breastfeeding during pregnancy induces diarrhea for infants" [Mother 8 Dagahle, Gursum woreda].

"I provide the complementary food at the same time with other household. There is no special time for child to be fed alone" [Mother 9, Dagahbur woreda].

\subsubsection{Women's Workload (Pattern and Burden of Other Responsibilities)}

Women's workload required them to either tend to crop cultivation, livestock rearing or fetching water on daily basis. Often, they left their children for hours in the care of other children, grandmothers, neighbors, or family members who commonly offered milk, Fax or food to calm the child when crying. This was a frequently cited reason for early introduction of complementary foods. Although not a widely adopted practice, there were a few women across the study sites who reported carrying their child with them to the field in order to practice exclusive breastfeeding. There were mixed responses from women as to whether they felt they could take their children with them to the field. Factors such as distance to the field, conditions in the field, and the load the women have to carry were reported as concerns.

"I am pastoralist, we have number of livestock, some of these livestock are left at home, and others are kept by other members of the family, livestock left at home need water and pasture. We are here living in an area with chronic water shortage where you should walk long distance to fetch water for the animal and household, leaving the child with relatives. If the child cries they give what is available, Fax, milk or food even for those less than six months" [Mother 5, Sanmadede, Goljano, FGDs].

\subsubsection{Poor Maternal Dietary Intake and Perceived Milk Insufficiency}

Mothers attributed their inability to produce enough milk to lack of access or availability of sufficient food in the household. Some participants indicated that they do household chores without food the whole day except consuming some water and a piece of Injera or Shuro with no milk. They tend to link their inability to produce breastmilk to this scenario in the household. Some participants also reported their child cries during or after feeding, and this leads them to think that their milk production is insufficient.

"We have lost our livelihood due to 2016/17 drought and have no livestock for milking and we are dependent on government support which is fluctuating. Therefore, if I am not getting enough food, sure, breastmilk is not enough for the baby, I fear for my baby, I look for additional food or milk from neighbors or relatives" [Mother 6, Agasur, Dagahbur woreda, FGD].

Some care givers also believe that breast milk alone is insufficient for the child and it needs to be supplemented with other foods including; baby formula, sugar water and animal milk. Water is also provided to quench the thirst of young children, particularly in the dry and hot seasons, as depicted below. 
"Breastmilk only is not enough for the infants, so, I provide animal milk, water should also be provided to satisfy the thirst for the infants" [Mother, Goljano 5, Goljano].

\subsubsection{Culture Custodian Influence (on Initiation, Exclusive and Complementary Feeding)}

Our study revealed that mothers give their infants holy water (taxalil), this holy water is believed to prevent children from evil eye, in addition this, the study also identified that mothers give their infants digri (herbal infusion), this herbal is believed to treat disease called "masreeye"; which prevent infant to properly grow.

"Let's say digri is a leave, I put it in a cup with small water, I put a little so that it sorts of soaks and then give this to the baby to drink in the morning, afternoon and evening" [Mother 6, sandmadhedhe, Goljano, FGD].

This practice of giving a baby water and herbal infusions is reinforced by important and influential members of the family, such as grandmothers echoed by FGDs.

"Mothers give the baby digri to drink as prevention of masreeye and I don't see anything wrong with this. I encourage giving dirgri; this assures infants to grow strong, as they are protected' [Grandmother 5, sandmadhedhe, Goljano, $F G D$.

Another traditional belief on initiation of breastfeeding in the study area is giving sugary water immediately after delivery; this sugary water is given to infants to stimulate appetite as revealed by the study participants. Giving sugary water as pre-lacteal feeding is also recommended by influential members of the family like, grandmothers. Besides, our study also revealed that fathers were not aware of what is given to their infants. Fathers consider this as women's role.

"I provide sugary water to infants immediately after delivery. I give this sugary water to stimulate appetite; the infant starts the breastmilk and other liquids with high appetite" [Mothers, Dhagahbur 4, and FGD].

"Production of breastmilk takes at least three days and the infant needs milk and what to drink, so, I recommend watery sugar to be given for survival' [Grandmother 3, Agasur, Dhagahbur, FGD].

"Roles are divided in the family, women are involved in infant and child feeding, and so, we are not aware of what mothers are giving the infants" [Father 4, Goray, Gursum, FGD].

The desire for a baby to grow up strong and healthy is critical to encourage breastfeeding in Somali communities. Religion is also an important influence on breastfeeding practices in Somali, as the Quran's support for breastfeeding is highlighted by religious leaders. In this survey, some religious leaders were interviewed on initiation of breastfeeding, particularly giving holy water to infants immediately after delivery. We have revealed mixed responses from the religious leaders at villages. The study team decided to move the question on giving holy water to infants to other religious leaders in the region. As result of this, we 
identified that there is no solid evidence from authentic Hadith or any other sources which encourage holy water to be given to infants immediately after delivery.

"Holly water can be given to infants immediately after delivery" [Religious leader 3, Dagahle, Gursum, interview].

"There is no evidence from authentic Hadith (sayings of the Prophet $\mathrm{Mu}$ hammad) or any other sources for holy water to be given to infants immediately after delivery. But small piece of dates (timir) is usually indicated to be put into infant's mouth immediately after delivery. I think the holy water is used in the absence of dates (timir) at these villages, if this holy water is used with Quran properly that will benefit" [Religious leader, Jigjiga, interview].

\section{Discussion}

The findings provide insight into the optimal maternal and child feeding practices, focusing on barriers for maternal and child feeding in Somali region. The results of the present study show that there are seven barriers including knowledge, myths and misconceptions, influence of culture custodians, and patterns, burden of household chores, accessibility of health facilities, poor maternal dietary intake and perceived breastmilk insufficient as the main barriers affecting appropriate maternal and child-feeding practices.

The respondents, particulars primary caregivers and religious leaders lacked knowledge about optimal maternal nutrition during pregnancy and lactation. Lack of knowledge about aspects, such as increased requirement of nutrients or food during pregnancy, caregivers have also low knowledge when it comes to type of diet to eat, mothers had low knowledge level about optimal diets needed during pregnancy. Pregnant mothers had information only that animal source foods are needed during pregnancy, but, they do not know other components of diets needed during pregnancy such as fruits, legumes and vegetables.

The participants mentioned that animal foods are very nutritious if available but provided less importance for fruits, legumes and vegetables at this period, this was one of the indicators of low knowledge on diversified foods.

The finding is comparable with research conducted in Northeastern Nigeria which revealed that mothers lack of knowledge of the physiological state of pregnancy and the fetal development process in relation to maternal diet, type of nutrients to take during pregnancy, lactation and the need for greater food consumption during certain life stages [8].

In addition to this, lack of knowledge is also identified as barrier for optimal child feeding practices. Majority of the participants in the FGDs reported that they gave holy water and fax to children immediately after delivery, until breastmilk comes to the breast freely at least after three days, the study participants indicated that they gave fax for these three days.

Furthermore, consistent with our finding, other research in Somali community and other regions in Ethiopia have found that mothers or caregivers educa- 
tion has effect on appropriate nutrition practice [40]. Similar study in Egypt also reported pregnant women have little to no knowledge of optimal weight gain during pregnancy, likely due to lack of counseling by health care providers [44].

Poor knowledge is also reported in research conducted Burkina Faso in which poor knowledge on some nutrients led women to restrict taking optimal diets [45]. Furthermore, poor knowledge as barrier for optimal maternal infants and young child nutrition also reported in other developing countries [31] [32] [33] [34] [35] [46].

Lack of knowledge may be impacting critical aspects of maternal and childfeeding, such as adequate maternal nutrition, early initiation of breastfeeding, exclusive breastfeeding for six months, and timely introduction of age appropriate complementary feeding, all of which are key aspects of the process of preventing maternal and childhood malnutrition and associated mortality. Therefore, maternal, infant and young child feeding strategy which is currently implemented by government of Ethiopia and partners need strengthening in particular at community level. The present study also revealed myths, misconception and culture custodian as barriers for optimal maternal and child nutrition practice, these myths and misconceptions include, mother's belief on association of pregnancy with diseases and difficulties, decrease of intake to prevent fetus to become larger than birth canal and limiting intake of Iron supplementation to prevent caesarian session.

This misconception of limiting intake of Iron supplementation and nutritious foods is also recommended by influential members in the households, such as grandmothers. The primary caregivers were following recommendations and information given by influential members in the family or community whom they respected because of their perceived understanding about cultural issues, traditions, and health matters to guide their maternal nutrition practice.

Misconception related to Iron intake is also reported in Egypt in which mothers threw Iron pills given by doctors [44].

This study also revealed a number of myths and misconception as barriers to optimal infant and child feeding practices includes giving infants holy water (Taxalil). This holy water is believed to prevent children from evil eye. Furthermore, the study also reported that mothers give their infants Digri (herbal infusion) and sugary water (Fax).

Digri and Fax were reported to be a major problem during infancy, and it interfered with initiation and exclusive breastfeeding practices. DigriIn is herbal infusion believed to treat colic related problem called "masreeye". Mothers believe this herbal infusion prevent disease and support infant to properly grow. Similarly, primary caregivers also provide fax (sugary water) as prelactal feeding. Studies in the United Arab Emirates, India, and Nigeria report mothers gave prelacteal feeds and gripe water to manage infant colic [47].

Findings related to our finding are also reported in Nigeria which revealed that mothers attributed the child's health to "divine will [8]. The result is also 
comparable to research conducted in Ethiopia which reported that women "think that the health of the baby is determined by God". (9). Other studies which are similar to this finding also cited beliefs that the fetus and food were both located in the stomach, and therefore if women ate too much food the baby would not have room to grow [10].

Myths on infant feeding practice are also reported in other parts of Ethiopia in which mothers believe health of the fetus is determined by God but not food taken by mothers [45]. In addition to this, although the type and context are different, misconceptions and perceptions as barriers are also reported in studies conducted in Zimbabwe [31] [33], Uganda [32], other developing countries [45], India [35] and Vietnam [48].

Access and affordability of different food types is another barrier for mothers to have fully diversified nutrients needed during pregnancy and lactation. Pastoralist communities in the study area such as villages under Dagahbur and Goljano woredas have poor access to different food types that are needed during pregnancy, lactation and complementary age. Even where nutritious foods were available for consumption, accessing them was hindered by lack of affordability and other household-level factors. Foods perceived to maximize pregnancy outcomes were often unaffordable, preventing pregnant women from supplementing their traditional diets with nutrient-rich foods.

This finding is in line with research conducted in Gozamin, Northern Eastern Ethiopia which reported affordability as barrier indicating families do not have the ability to buy various types of fruits, vegetables and cereals, so families just prepare food from what is available at hand [37]. Accessibility and poverty as barrier was also reported in researches conducted in Bangladesh [34], Loas [49], Northeast Nigeria [8], Egypt [50], Ethiopia [9], India [35] and Tanzania [12]. Lack of access to nutritious foods or economic constraints is also reported as major barrier to obtaining food in Indonesia, Burkina Faso, India, Kenya, Nepal, Nigeria and Pakistan [12].

Our study further highlighted high burden of other responsibilities as barrier for optimal nutrition, pastoralist women are busy with livestock rearing and household chores, the primary caregivers hinted on a very high burden of household chores, and these tended to intensify during dry season. Participants indicated negative perception culturally if the husband supports pregnant women for household activities; the primary caregivers received limited to no support from the male adults (or husbands) in the family towards meeting the maternal, infant and young child-feeding needs or reducing the burden of household chores and other responsibilities.

This finding is similar with research conducted in Northern eastern Ethiopia, which states that mothers/caretakers did not properly feed their children due to different workload from outside and inside the home [37]. Consistent results were also reported from other studies conducted in Uganda [32], Congo [36], Zambia [51], and Ethiopia [37], and Bangladesh [34], Thailand [52], Zimbabwe [31]. 
The other major barrier for optimal child feeding practice was poor maternal dietary intake and perceived milk insufficiency, perceived insufficient breast milk production emerged as one of the barriers, particularly, to exclusive breastfeeding (EBF). This perception is one reason why mothers introduced their babies to other foods earlier than recommended. In addition to this, participants also indicated poor maternal dietary intake reduce amount of breastmilk, this also another reason for pastoralist mother introduced their babies to other foods particularly during dry season when availability of foods and dairy products decreases, as result of this, mothers in the study area traditionally give infants sugary water (fax) before six months. Similar finding is reported in Kenyan pastoralist community; barrier to EBF from birth to 6 months is the participants' belief that their milk alone is insufficient nutrition for their infant [53]. Similarly, Webb-Girard et al. found food insecure women in Kenya were significantly more likely to believe their milk was insufficient [54]. Another finding from Congo in which most women reported their child cried during or after feeding, and this leads them to think that their milk production was insufficient [36]. The same results were also reported from studies in Zimbabwe [33], Ghana [38], and Tanzania [39].

\section{Conclusion}

This research has provided valuable local insight regarding the maternal child feeding among care givers and key informants who are source of advice on cultural issues relating to nutrition practices. Their descriptions and insight revealed barriers on optimal maternal and child feeding practice as recommended by the WHO, particularly caregivers of children between $0-23$ months. The result of the study indicates more work needs to be undertaken to strengthen community-based nutrition with strong social and behavioral change communication. Emphasizing on age-specific counselling on maternal infant and young child nutrition at health facilities during antenatal, early postnatal visits and outreach services in the pastoralist areas through cultural sensitive multi-channel media for instance, SRTV and FMs is critical to promote optimal nutrition in Somali region and reverse prevalent harmful feeding and caring practices with far-reaching negative consequences on maternal and child health.

\section{Limitation}

This is a qualitative study, and the sample was not representative of the entire population of primary caregivers, grandmothers, grandfathers, religious leaders and health professionals in the districts and did not include other people in the community. The districts where the study was conducted were pastoralist, therefore, other livelihood zones in Somali region, like riverine livelihood were not included. Despite the limitations of this study, the findings provide a starting point for serial interventions to improve maternal, infants and young child nutrition in the study area. 


\section{Acknowledgements}

We are very grateful to the Jigjiga University for financial support of this study. We are also very grateful and extend our appreciation to the study participants who shared their crucial time, the supervisors, and the data collectors for their full commitment for participation and technical support.

\section{Conflicts of Interest}

The authors declare no conflicts of interest regarding the publication of this paper.

\section{References}

[1] Bhutta, Z.A., Ahmed, T., Black, R.E., et al. (2013) What Works? Interventions for Maternal and Child Undernutrition and Survival. The Lancet, 371, 417-440.

https://doi.org/10.1016/S0140-6736(07)61693-6

[2] Barker, M., Dombrowski, S.U., Colbourn, T., Fall, C.H.D., Kriznik, N.M., Lawrence, W.T., et al. (2018) Intervention Strategies to Improve Nutrition and Health Behaviours before Conception. The Lancet, 391, 1853-1864. https://doi.org/10.1016/S0140-6736(18)30313-1

[3] Shrimpton, R. (2012) Global Policy and Programme Guidance on Maternal Nutrition: What Exists, the Mechanisms for Providing It, and How to Improve Them. Paediatric and Perinatal Epidemiology, 26, 315-325. https://doi.org/10.1111/j.1365-3016.2012.01279.x

[4] Haddad, L., Cameron, L. and Barnett, I. (2015) The Double Burden of Malnutrition in SE Asia and the Pacific Priorities, Policies and Politics. Health Policy and Planning, 30, 1193-1206. https://doi.org/10.1093/heapol/czu110

[5] Black, R.E., Allen, L.H., Bhutta, Z.A., et al. (2008) Maternal and Child Undernutrition: Global and Regional Exposures and Health Consequences. The Lancet, 371, 243-260.

[6] Lakshmi, G. (2013) Food Preferences and Taboos during Ante-Natal Period among the Tribal Women of North Coastal Andhra Pradesh. Journal of Community Nutrition \& Health, 2, 32-37.

[7] Choudhury, N., Moran, A.C., Alam, M.A., Ahsan, K.Z., Rashid, S.F. and Streatfield, P.K. (2012) Beliefs and Practices during Pregnancy and Childbirth in Urban Slums of Dhaka, Bangladesh. BMC Public Health, 12, Article No.: 791. https://doi.org/10.1186/1471-2458-12-791

[8] Girard, A.W., Dzingina, C., Akogun, O., Mason, J.B. and McFarland, D.A. (2012) Public Health Interventions, Barriers, and Opportunities for Improving Maternal Nutrition in Northeast Nigeria. Food and Nutrition Bulletin, 33, S51-S70. https://doi.org/10.1177/15648265120332S104

[9] Saldanha, L.S., Buback, L., White, J.M., Mulugeta, A., Mariam, S.G., et al. (2012) Policies and Program Implementation Experience to Improve Maternal Nutrition in Ethiopia. Food and Nutrition Bulletin, 33, S27-S50. https://doi.org/10.1177/15648265120332S103

[10] Huybregts, L.F., Roberfroid, D.A., Kolsteren, P.W. and Van Camp, J.H. (2009) Dietary Behaviour, Food and Nutrient Intake of Pregnant Women in a Rural Community in Burkina Faso. Maternal \& Child Nutrition, 5, 211-222. https://doi.org/10.1111/j.1740-8709.2008.00180.x 
[11] Oni, O.A. and Tukur, J. (2012) Identifying Pregnant Women Who Would Adhere to Food Taboos in a Rural Community: A Community-Based Study. African Journal of Reproductive Health, 16, 68-76.

[12] Young, A.G. and Pike, I.L. (2012) A Biocultural Framework for Examining Maternal Cravings and Aversions among Pastoral Women in East Africa. Ecology of Food and Nutrition, 51, 444-462. https://doi.org/10.1080/03670244.2012.696013

[13] PAHO/WHO (2003) Guiding Principles for Complementary Feeding of the Breastfed Child. PAHO/WHO, Washington DC.

[14] LANCET (2013) Maternal and Child Nutrition. Building Momentum for Impact. LANCET, 4, 15-39.

[15] Organization for Economic Co-operation and Development (OECD) World Health Organization (2018) Health at a Glance: Asia/Pacific. WHO, OECD Publishing, Tokyo.

[16] WHO (2009) Infant and Young Child Feeding: Model Chapter for Textbooks for Medical Students and Allied Health Professionals. WHO, Geneva, 41.

[17] Victora, C.G., Adair, L., Fall, C., Hallal, P.C., Martorell, R., Richter, L.M., et al. (2008) Maternal and Child Undernutrition: Consequences for Adult Health and Human Capital. The Lancet, 371, 340-357. https://doi.org/10.1016/S0140-6736(07)61692-4

[18] Ethiopia, Ministry of Health (2014) Health Policy and Practice, Information for Action. Quarterly Health Bull., Addis Ababa, 26-34.

[19] Alive \& Thrive (2011) An Advocate's Guide for Helping Advance Infant and Young Child Nutrition in Ethiopia. Alive \& Thrive, Washington DC.

[20] UNICEF (2015) State of the World's Children 2015 Country Statistical Information. UNICEF, New York, 42-47.

[21] Skeel, L.S. and Good, M.E. (1988) Mexican Cultural Beliefs and Breastfeediing: A Model for Assessment and Intervention. Journal of Human Lactation, 4, 160-163. https://doi.org/10.1177/089033448800400409

[22] Shirima, R., Greiner, T., Kylberg, E. and Gebre-Medhin, M. (2001) Exclusive BreastFeeding Is Rarely Practised in Rural and Urban. Public Health Nutrition, 4, 147-154. https://doi.org/10.1079/PHN200057

[23] Hizel, S., Ceyhun, G., Tanzer, F. and Sanli, C. (2006) Traditional Beliefs as Forgotten Influencing Factors on Breast-Feeding Performance in Turkey. Saudi Medical Journal, 27, 511-518.

[24] Wanjohi, M., Griffiths, P., Wekesah, F., et al. (2016) Sociocultural Factors Influencing Breastfeeding Practices in Two Slums in Nairobi. International Breastfeeding Journal, 12, Article No.: 5. https://doi.org/10.1186/s13006-016-0092-7

[25] Diji, A.K.-A., Bam, V., Asante, E., Lomotey, A.Y., Yeboah, S. and Owusu, H.A. (2017) Challenges and Predictors of Exclusive Breastfeeding among Mothers Attending the Child Welfare Clinic at a Regional Hospital in Ghana: A Descriptive Cross-Sectional Study. International Breastfeeding Journal, 12, Article No.: 13. https://doi.org/10.1186/s13006-017-0104-2

[26] Daglas, M. and Antoniou, E. (2012) Cultural Views and Practices Related to Breastfeeding. Health Science Journal, 6, 353-361.

[27] Kimani-Murage, E.W., Wekesah, F., Wanjohi, M., et al. (2015) Factors Affecting Actualisation of the WHO Breastfeeding Recommendation in Urban Poor Settings in Kenya. Maternal \& Child Nutrition, 11, 314-332. https://doi.org/10.1111/mcn.12161

[28] Vyas, S., Kandpal, S.D., Semwal, J., Chauhan, S. and Nautiyal, V. (2010) Trends in 
Weaning Practices among Infants and Toddlers in a Hilly Terrain of a Newly Formed State of India. International Journal of Preventive Medicine, 5, 741-748.

[29] Malhotra, N. (2013) Inadequate Feeding of Infant and Young Children in India: Lack of Nutritional Information or Food Affordability? Public Health Nutrition, 16, 1723-1731. https://doi.org/10.1017/S1368980012004065

[30] Chaturvedi, S., Ramji, S., Arora, N.K., Rewal, S., Dasgupta, R. and Deshmukh, V. (2016) Time-Constrained Mother and Expanding Market: Emerging Model of Under-Nutrition in India. BMC Public Health, 16, Article No.: 632. https://doi.org/10.1186/s12889-016-3189-4

[31] Maxwell, M., Mukora-Mutseyekwa, F.N.N., Zvinavashe, M. and Haruzivishe, C. (2015) Perceptions and Barriers to Exclusive Breastfeeding in Manicaland, Zimbabwe. Journal of Public Health Medicine, 21, 30.

[32] Nankumbi, J. and Muliira, J.K. (2015) Barriers to Infant and Child-Feeding Practices: A Qualitative Study of Primary Caregivers in Rural Uganda. Journal of Health, Population and Nutrition, 33, 106-116.

[33] Nduna, T., Marais, D. and van Wyk, B. (2015) An Explorative Qualitative Study of Experiences and Challenges to Exclusive Breastfeeding among Mothers in Rural Zimbabwe. Childhood Obesity and Nutrition, 7, 69-76. https://doi.org/10.1177/1941406414568562

[34] Hackett, K.M., Mukta, U.S., Jalal, C.S.B. and Sellen, D.W. (2015) A Qualitative Study Exploring Perceived Barriers to Infant Feeding and Caregiving among Adolescent Girls and Young Women in Rural Bangladesh. BMC Public Health, 15, Article No.: 771. https://doi.org/10.1186/s12889-015-2115-5

[35] Geethalakshmi, R.G. and Smitha Yadav, J.S. (2017) Qualitative Exploration of Infant and Young Child Feeding Practices in Rural Field Practice Area of SSIMS and RC: A Focus Group Discussion Study. International Journal of Community Medicine and Public Health, 4, 2787-2792. https://doi.org/10.18203/2394-6040.ijcmph20173324

[36] Burns, J., Emerson, J.A., Amundson, K., Doocy, S., Caulfield, L.E. and Klemm, R.D.W. (2016) A Qualitative Analysis of Barriers and Facilitators to Optimal Breastfeeding and Complementary Feeding Practices in South Kivu, Democratic Republic of Congo. Food and Nutrition Bulletin, 37, 119-131. https://doi.org/10.1177/0379572116637947

[37] Mekonnen, N., Asfaw, S., Mamo, A., Mulu, Y. and Fentahun, N. (2018) Barriers and Facilitators of Child-Feeding Practice in a Small Sample of Individuals from Gozamin District, Northwest of Ethiopia: A Qualitative Study. BMC Nutrition, 4, Article No.: 25. https://doi.org/10.1186/s40795-018-0233-Z

[38] Otoo, G.E., Lartey, A.A. and Pérez-Escamilla, R. (2009) Perceived Incentives and Barriers to Exclusive Breastfeeding among Periurban Ghanaian Women. Journal of Human Lactation, 25, 34-41. https://doi.org/10.1177/0890334408325072

[39] Mgongo, M., Hussein, T.H., Stray-Pedersen, B., Vangen, S., Msuya, S.E. and Wandel, M. (2019) Facilitators and Barriers to Breastfeeding and Exclusive Breastfeeding in Kilimanjaro Region, Tanzania: A Qualitative Study. International Journal of Pediatrics, 10, Article ID: 8651010. https://doi.org/10.1155/2019/8651010

[40] Central Statistical Agency (2016) Ethiopian Demographic and Health Survey (EDHS). CSA, Addis Ababa, 5.

[41] Sadler, K., Kerven, C., Calo, M., Manske, M. and Catley, A. (2009) Milk Matters: A Literature Review of Pastoralist Nutrition and Programming Responses. Feinstein International Center, Tufts University and Save the Children, Addis Ababa. 
[42] Central Statistical Agency (2011) Ethiopian Demography and Health Survey (EDHS). CSA, Addis Ababa, 32

[43] Braun, V. and Clarke, V. (2006) Using Thematic Analysis in Psychology. Qualitative Research in Psychology, 3, 77-101. https://doi.org/10.1191/1478088706qp063oa

[44] Kavle, J., Mehanna, S., Khan, G., Hassan, M., Saleh, G. and Galloway, R. (2014) Cultural Beliefs and Perceptions of Maternal Diet and Weight Gain during Pregnancy and Postpartum Family Planning in Egypt. USAID, Maternal and Child Health Integrated Program, 9.

[45] Kavle, J.A. and Landry, M. (2017) Addressing Barriers to Maternal Nutrition in Low- and Middle-Income Countries: A Review of the Evidence and Programme Implications. Maternal \& Child Nutrition, 14, e12508.

https://doi.org/10.1111/mcn.12508

[46] Beglay, A. (2002) Barrier to Good Nutrients Intake during Pregnancy: Qualitative Analysis. Nutrition \& Dietetics, 59, 175-180.

[47] Radwan, H. and Sapsford, R. (2016) Maternal Perceptions and Views about Breastfeeding Practices among Emirati Mothers. Food and Nutrition Bulletin, 37, 73-84. https://doi.org/10.1177/0379572115624289

[48] Lundberg, P.C. and Trieu, T.N. (2011) Vietnamese Women's Cultural Beliefs and Practices Related to the Postpartum Period. Midwifery, 27, 731-736. https://doi.org/10.1016/j.midw.2010.02.006

[49] Sa, J., Bouttasing, N., Sampson, L., Perks, C., Osrin, D. and Prost, A. (2013) Identifying Priorities to Improve Maternal and Child Nutrition among the Khmu Ethnic group: Formative Research. Maternal and Child Nutrition, 9, 452-466. https://doi.org/10.1111/j.1740-8709.2012.00406.x

[50] Kavle, J.A., Mehanna, S., Saleh, G., Fouad, M.A., Ramzy, M., Hamed, D., et al. (2014) Exploring Why Junk Foods Are "Essential" Foods and How Culturally Tailored Recommendations Improved Feeding in Egyptian Children. Maternal \& Child Nutrition, 11, 346-370. https://doi.org/10.1111/mcn.12165

[51] Ministry of Health (2010) Qualitative Assessment of Maternal Nutrition Practices in Zambia. The Infant \& Young Child Nutrition (IYCN) Project, Lusaka.

[52] Thepha, T., Marais, D., Bell, J. and Muangpin, S. (2017) Facilitators and Barriers to Exclusive Breastfeeding in Thailand: A Narrative Review. Journal of Community and Public Health Nursing, 3, 160. https://doi.org/10.4172/2471-9846.1000160

[53] Leurer, M.D., Petrucka, P. and Msafiri, M. (2019) Maternal Perceptions of Breastfeeding and Infant Nutrition among a Select Group of Maasai Women. BMC Pregnancy and Childbirth, 19, Article No.: 8. https://doi.org/10.1186/s12884-018-2165-7

[54] Webb-Girard, A., Charobon, A., Mbugua, S., Kamau-Mbuthia, E., Amin, A. and Sellen, D.W. (2010) Food Insecurity Is Associated with Attitudes towards Exclusive Breastfeeding among Women in Kenya. Maternal \& Child Nutrition, 8, 199-214. https://doi.org/10.1111/j.1740-8709.2010.00272.x 